\title{
Situação no mercado de trabalho e utilização de serviços de saúde no Brasil
}

\author{
Labor market status and the use of healthcare services in Brazil
}

Luana Giatti ${ }^{1}$

Sandhi Maria Barreto ${ }^{1}$
${ }^{1}$ Programa de PósGraduação em Saúde Pública, Faculdade de Medicina, Universidade Federal de Minas Gerias. Av Alfredo Balena 190/814, Santa Efigênia. 30190-100 Belo Horizonte MG.luana.giatti@gmail.com
Abstract This study compares the use of health services among economically active men according to labor market status in the years 1998, 2003, 2008 and investigates whether both are associated after adjustment for socio-demographic characteristics and health indicators. All males aged 15 to 64, participating in the National Household Survey 1998, 2003 and 2008 were included. The association between labor market status and use of services in 2008 was estimated by Poisson regression. 33,726 males were surveyed and the prevalence of medical and dental care use was found to be higher among workers with social protection. The frequency of health care use increased over the period, but remained significantly higher among formally employed workers. Unemployment and work without social protection were negatively associated with medical and dental care visits. The socio-economic structure that establishes different social groups with unequal health conditions also affects the use of health services. Further investigation into the social and physical barriers to health care use by the unemployed and informal workers is required in order to reduce perceived health inequities.

Key words Unemployment, Precarious work, Use of healthcare services, Healthcare inequalities
Resumo Este estudo compara a utilização de serviços de saúde segundo a situação no mercado de trabalho em três anos e investiga se estão associados após ajuste por características sócio-demográficas e presença de doença crônica. Foram estudados homens de 15 a 64 anos, incluidos na PNAD de 1998, 2003 e 2008 de acordo com a utilização de consulta médica e internação nos últimos 12 meses e visita ao dentista há menos de três anos. A associação entre a situação no mercado de trabalho e o uso de serviços de saúde em 2008 foi estimada pela regressão de Poisson. Foram estudados 33.726 homens. As prevalências de consulta médica e odontológica aumentaram ao longo do período, mas mantiveram-se mais elevadas entre os trabalhadores com proteção social. Desemprego e trabalho sem proteção social foram independente e negativamente associados à menor prevalência de consulta médica e odontológica em 2008. Conhecer as barreiras e aumentar a oferta de serviços com perfil de funcionamento adequado para quem trabalha é fundamental para tentar reduzir as iniquidades observadas. Por outro lado, promover a inserção no trabalho com garantias dos direitos sociais pode contribuir para a melhoria dos indicadores de saúde pública.

Palavras-chave Desemprego, Trabalho precário, Uso de serviços de saúde, Desigualdades em saúde 


\section{Introdução}

As desigualdades em saúde se expressam em diferentes dimensões desta e em aspectos da posição social dos indivíduos e dos grupos sociais, incluindo o trabalho. O grupo de trabalho da Comissão de Determinantes Sociais da Saúde da OMS, Employment Conditions Knowledge Network (EMCONET)2007, produziu um importante relatório analisando como as relações de emprego afetam a saúde dos grupos da população e como esse conhecimento pode identificar e promover políticas efetivas e mudanças institucionais para reduzir as desigualdades em saúde provenientes das relações de emprego. Esse estudo abrange o contexto político, econômico e cultural para produzir um amplo retrato da situação do mercado de trabalho e das condições de emprego em todo o mundo ${ }^{1}$.

Em recente comentário publicado na revista American Journal of Industrial Medicine, Krieger ${ }^{2}$ reforça a necessidade de se considerar que homens e mulheres, de diferentes etnias, com diferentes níveis de escolaridade, em diferentes contextos, que estejam empregados, inseridos em um ou outro tipo de trabalho, sob diferentes exposições, com ou sem estabilidade, com ou sem benefícios ou desempregados estão sob condições que influenciam suas vidas e a saúde simultaneamente.

Os estudos realizados em diversos países, inclusive no Brasil, mostram que há considerável evidência de que o desemprego esteja associado à maior mortalidade e morbidade $e^{3-6}$, e à adoção de comportamentos que podem influenciar negativamente a saúde $e^{7,8}$. Por outro lado, também se reconhece que condições de trabalho inadequadas, atuais e passadas, em seus aspectos físicos, organizacionais e psicossociais contribuem com as desigualdades em saúde ${ }^{9-11}$. Na atualidade, ganha importância o papel dos estressores psicossociais no ambiente de trabalho e sua relação com doenças como hipertensão e outras cardiovasculares nos trabalhadores de menor nível socioeconômico ${ }^{12}$.

Devido às mudanças socioeconômicas ocorridas nas últimas décadas, houve o avanço de novas modalidades de inserção no mercado laboral, como o trabalho temporário ou sem contrato que, em geral, apresenta instabilidade, menores salários e piores condições no exercício da profissão. Essas situações, heterogeneamente distribuídas entre os trabalhadores, conferem novos riscos à saúde e podem contribuir com as desigualdades sociais neste campo ${ }^{12,13}$. Os achados indicam que adultos com inserção em for- mas de trabalho precário apresentam indicadores de posição socioeconômica e condições de saúde piores do que aqueles que têm estabilidade profissional. Estudo realizado no Brasil observou que indivíduos inseridos em trabalhos sem proteção social, expostos a instabilidade e vulnerabilidade, apresentavam menores níveis de escolaridade, de renda e piores condições de saú$\mathrm{de}^{14}$. Na Coreia do Sul, um estudo de painel mostrou que trabalhadores inseridos em trabalhos precários apresentavam menor escolaridade, menor renda domiciliar, atuavam mais frequentemente em atividades manuais do que os inseridos em trabalhos não precários e também mostraram maior probabilidade de avaliar sua saúde como ruim ${ }^{15}$. No Canadá, trabalhadores com contratos temporários estavam mais propensos a apresentar sintomas depressivos ${ }^{16}$. Já um estudo realizado na Finlândia sugere que trabalhadores com contratos temporários não constituem um grupo homogêneo; pois somente aqueles inseridos em situações instáveis e que relatavam insatisfação com a ocupação ou os que estavam involuntariamente em trabalhos temporários apresentaram maior risco de mortalidade do que os trabalhadores com contratos permanentes ${ }^{17}$.

As desigualdades em saúde em grupos sociais distintos, que refletem a distribuição desigual de recursos e oportunidades, são consideradas injustas $^{18}$, desnecessárias e evitáveis, e por isso chamadas de iniquidades. Com o intuito de diminuir as diferenças sociais, as políticas públicas podem atuar sobre os diversos níveis da determinação social das desigualdades em saúde, seja por meio da educação, da ampliação da proteção social ou da redistribuição de renda. Outras políticas públicas, como as ações dos sistemas de saúde, podem intervir sobre os diferenciais de vulnerabilidades ${ }^{19,20}$. No Brasil a saúde é um direito de todos os cidadãos e a garantia de acesso universal aos seus cuidados se dá por meio do Sistema Único de Saúde (SUS). Entretanto, estudos mostram importantes desigualdades relacionadas à distribuição e utilização dos serviços de saúde no país.

Diferenças na utilização dos serviços de saúde podem refletir necessidades distintas entre os indivíduos, que variam segundo a idade, o sexo e as condições de saúde ${ }^{21}$. Entretanto, quando refletem disparidades na disponibilidade de serviços entre grupos sociais distintos e são influenciadas por características socialmente determinadas como escolaridade, renda e trabalho, estas diferenças são consideradas inaceitáveis e contribuem para explicar desigualdades sociais em indicadores gerais como adoecimento e morte. 
O presente estudo pretende comparar a utilização de serviços de saúde em homens economicamente ativos segundo a situação no mercado de trabalho nos anos de 1998, 2003 e 2008, e investigar se ambos estão associados após ajuste por características sócio-demográficas e por um indicador de necessidade de saúde. A população de estudo se restringiu aos homens porque há reconhecido diferencial de gênero no adoecimento, percepção de saúde e utilização dos serviços ${ }^{22}$ e na inserção no mercado de trabalho ${ }^{23}$. Homens, em especial aqueles em idade ativa, a despeito da maior risco de adoecimento e morte, utilizam menos os serviços de saúde do que as mulheres.

\section{Métodos}

Este estudo utilizou dados da Pesquisa Nacional por Amostra de Domicílios (PNAD) realizada pela Fundação Instituto Brasileiro de Geografia e Estatísticas. Anualmente, a PNAD obtém informações sobre características sócio-demográficas, educacionais, de trabalho, dentre outras; nos anos 1998, 2003 e 2008, foram acrescentados suplementos de saúde.

Para essa análise, foram incluídos homens com idade de 15 a 64 anos, que faziam parte da população economicamente ativa e residiam nas regiões metropolitanas de Fortaleza, Recife, Salvador, Belo Horizonte, Rio de Janeiro, São Paulo, Curitiba e Porto Alegre. As regiões metropolitanas da região norte (Belém) e Centro-Oeste (Brasília) forma excluídas por apresentarem características no mercado de trabalho muito distintas das demais

A PNAD utiliza uma amostra probabilística complexa obtida em dois ou três estágios de seleção. Foram incluídos no presente estudo apenas os municípios de regiões metropolitanas, onde a amostra é obtida em dois estágios. As unidades primárias de amostragem são os setores censitários e as unidades secundárias são os domicílios ${ }^{24}$.

\section{Variáveis estudadas}

A utilização dos serviços de saúde foi mensurada pelas variáveis consulta médica nos últimos 12 meses (nenhuma, uma ou mais), internação hospitalar nos últimos 12 meses (não, sim) e visita ao dentista nos últimos três anos (nenhuma, uma ou mais).

A variável explicativa de interesse foi a situação no mercado de trabalho, categorizada em trabalho com proteção social, trabalho sem pro- teção social e desemprego. Trabalho com proteção social corresponde ao trabalho com carteira assinada ou apenas com contribuição para previdência social. Trabalho sem proteção social é aquele sem carteira assinada e sem contribuição para previdência social. Desemprego corresponde à condição de indivíduos que não trabalhavam e procuraram por trabalho na semana de referência adotada pela PNAD.

As outras características sócio-econômicas inseridas foram escolaridade em anos completos de estudo (0-7, 8-10,11-14, 15+), renda domiciliar per capita agrupada em quintis, idade em faixas etárias (15-24, 25-34, 35-44, 45-54, 55-64 anos) e a região de residência (sudeste, sul e nordeste). O número de doenças crônicas (nenhuma, uma, duas ou mais) informado foi considerado para ajuste, pois é um indicador de necessidade de utilização de serviços de saúde. Esta variável foi obtida pela soma das respostas a perguntas que investigavam relato de diagnóstico médico das doenças artrite ou reumatismo, asma ou bronquite, câncer, diabetes, hipertensão, doença do coração, insuficiência renal crônica e depressão.

Para descrever algumas características de utilização dos serviços de saúde, foram acrescentadas as variáveis procura o mesmo serviço de saúde (sim, não) obtida pela pergunta Costuma procurar o mesmo lugar, mesmo médico ou mesmo serviço de saúde quando precisa de atendimento de saúde? e tipo de serviço que procura (centro de saúde, consultório particular, ambulatórios que incluem ambulatório ou consultório de empresa ou sindicato, ambulatório ou consultório de clínica e ambulatório ou consultório de hospital, emergências e outros que reúne farmácia, agente comunitário e outro tipo de serviço) obtida pela pergunta Quando está doente ou precisando de atendimento de saúde costuma procurar.... Além dessas, também incluímos a cobertura por plano de saúde ( $\operatorname{sim}$, não) e o responsável pelo pagamento do plano de saúde.

\section{Análise}

Inicialmente foram estimadas as prevalências e seus intervalos de confiança de 95\% de consulta médica nos últimos 12 meses, internação hospitalar nos últimos 12 meses e visita ao dentista nos últimos três anos segundo a situação no mercado de trabalho para os três anos.

As análises seguintes referiram apenas ao ano de 2008. Foi feita a descrição das prevalências de consulta médica e internação hospitalar nos últimos 12 meses e de visita ao dentista nos últimos 
três anos segundo demais características sóciodemográficas e, para consulta médica, de acordo com o número de doenças crônicas. A associação entre as variáveis respostas e as explicativas foi medida por meio do teste de qui-quadrado de Pearson com nível de significância de 0,05. Em seguida, utilizando-se regressão de Poisson com variância robusta, foi feita a análise univariada para obter a razão de prevalências e seu intervalo de confiança de 95\%. Por último, realizou-se a análise multivariada com a inclusão das variáveis que se apresentaram associadas à variável resposta com valor de $\mathrm{p} \leq 0,20$. A categoria de referência para a variável consulta médica nos últimos 12 meses foi não a ter realizado, para internação hospitalar nos últimos 12 meses foi não ter sito internado e para visita ao dentista nos últimos três anos foi não a ter feito.

Por fim, foi realizada a descrição das características de utilização dos serviços de saúde.

Para corrigir as diferentes probabilidades de seleção de cada indivíduo foram utilizados pesos na estimativa das prevalências. A análise foi feita no software Stata (versão 10.0) utilizando o procedimento "svy" (com fatores de ponderação) adequado para análises de dados obtidos por plano amostral complexo.

\section{Resultados}

Em todos os anos, a prevalência de consulta médica foi maior entre os trabalhadores que tinham proteção social. No período estudado, sua frequência aumentou entre os que trabalhavam com proteção social, sem proteção social e que estavam desempregados. A magnitude desses aumentos foi semelhante nos três grupos, mantendo-se, ao longo do período, praticamente a mesma discrepância observada em 1998 com relação à situação no mercado de trabalho. Nos três anos estudados, o item visita ao dentista nos últimos três anos foi menos frequente entre os trabalhadores sem proteção social. No período de estudo, houve aumento similar do percentual de homens que relataram visita ao dentista nos últimos três anos em todos os grupos analisados, permanecendo o diferencial no uso de serviços odontológicos segundo a situação no mercado de trabalho. Já a proporção de homens em cada uma das situações no mercado de trabalho que informaram internação hospitalar nos últimos 12 meses permaneceu praticamente inalterada nos três anos (Tabela 1 ).

No ano de 2008, foram analisados 33.726 homens que apresentaram uma média de idade de 36,3 anos, $70 \%$ residiam na região sudeste do país, $13,6 \%$ na região sul e 19,4\% na região nordeste. Entre eles, $62,7 \%$ trabalhavam com proteção social, 30,2\% estavam em ocupações sem proteção social e 7,1\% desempregados. Na Tabela 2, verifica-se que a maioria dos homens que trabalhavam com proteção social tinha entre 25 e 44 anos de idade e mais de 11 anos de estudos. Entre os que trabalhavam sem proteção social verificouse uma maior proporção de indivíduos na faixa etária mais velha e com menor escolaridade. Já entre os desempregados, predominavam os mais jovens e os que se encontravam no quintil de renda domiciliar per capita mais baixo.

A prevalência de consulta médica aumentou diretamente com a idade, a escolaridade, a renda

Tabela 1. Prevalência de consultas médicas, internação hospitalar e visita ao dentista segundo inserção no mercado de trabalho em homens residentes em oito regiões metropolitanas brasileiras, PNAD $1998,2003,2008$.

\begin{tabular}{|c|c|c|c|}
\hline & 1998\% (IC95\%) & $2003 \%($ IC95\%) & $2008 \%($ IC95\%) \\
\hline \multicolumn{4}{|c|}{ Consulta médica nos últimos 12 meses } \\
\hline Com proteção social & $52,5(51,6-53,3)$ & $57,3(56,5-58,2)$ & $61,9(61,2-62,8)$ \\
\hline Sem proteção social & $39,6(38,4-40,7)$ & $43,6(42,6-44,7)$ & $49,6(48,4-50,7)$ \\
\hline Desempregado & $40,9(38,9-42,9)$ & $42,7(40,8-44,5)$ & $50,4(48,1-52,7)$ \\
\hline \multicolumn{4}{|c|}{ Internação hospitalar nos últimos 12 meses } \\
\hline Com proteção social & $3,1(2,8-3,4)$ & $3,5(3,2-3,8)$ & $3,7(3,4-4,0)$ \\
\hline Sem proteção social & $2,9(2,5-3,3)$ & $3,0(2,8-3,4)$ & $3,2(2,9-3,9)$ \\
\hline Desempregado & $2,4(1,8-3,0)$ & $2,6(2,0-3,2)$ & $3,2(2,4-4,0)$ \\
\hline \multicolumn{4}{|c|}{ Visita ao dentista há menos de 3 anos } \\
\hline Com proteção social & $68,2(67,4-69,0)$ & $73,3(72,5-74,0)$ & $77,8(77,1-78,4)$ \\
\hline Sem proteção social & $52,1(51,0-53,3)$ & $56,6(55,6-57,7)$ & $61,2(60,1-62,3)$ \\
\hline Desempregado & $57,0(40,9-45,0)$ & $60,7(58,9-62,5)$ & $68,5(66,3-70,7)$ \\
\hline
\end{tabular}


domiciliar per capita, o número de doenças crônicas e foi mais elevada entre os moradores da região sul. $\mathrm{Na}$ análise univariada, a consulta médica esteve negativamente associado com trabalho sem proteção social e desemprego, e positivamente associada à idade, à escolaridade, à renda domiciliar per capita, com moradia na região sul e com número de doenças crônicas. Após o ajuste pelas demais variáveis sócio-demográficas e pelo número de doenças crônicas, apesar da atenuação nas razões de prevalência, o trabalho sem proteção social permaneceu negativamente associado à consulta médica, assim como o desemprego. Homens inseridos no trabalho sem proteção social apresentaram uma probabilidade $17 \%$ menor de ter realizado consulta médica nos últimos
12 meses do que aqueles inseridos no trabalho com proteção social (Tabela 3).

$\mathrm{Na}$ análise univariada, a situação no mercado de trabalho não foi estatisticamente associada à internação hospitalar nos últimos 12 meses $(\mathrm{p}=0,15)$. Foram procedidos os ajustes por variáveis sócio-demográficas e pelo número de doenças crônicas, sem alteração da significância estatística (dados não mostrados).

A prevalência de visita ao dentista nos últimos três anos diminuiu com a idade, aumentou com a escolaridade e com a renda, e foi maior entre os moradores da região sul. Visita ao dentista nos últimos três anos apresentou associação negativa com inserção no trabalho sem proteção social, com desemprego e com a idade. Es-

Tabela 2. Distribuição (\%) de homens residentes em oito regiões metropolitanas participantes da PNAD 2008 segundo a situação no mercado de trabalho, características sócio-demográficas e número de doenças crônicas.

\begin{tabular}{|c|c|c|c|}
\hline & \multicolumn{3}{|c|}{ Situação no mercado de trabalho } \\
\hline & Com proteção social & Sem proteção social & Desempregado \\
\hline \multicolumn{4}{|l|}{ Faixa etária } \\
\hline $15-24$ & 16,0 & 23,7 & 48,7 \\
\hline $25-34$ & 30,4 & 23,5 & 23,5 \\
\hline $35-44$ & 26,7 & 22,3 & 12,6 \\
\hline $45-54$ & 19,8 & 18,7 & 10,6 \\
\hline \multirow[t]{2}{*}{$55-64$} & 8,1 & 11,9 & 4,5 \\
\hline & & $\mathrm{p}<0,0001$ & \\
\hline \multicolumn{4}{|l|}{ Escolaridade } \\
\hline $0-3$ & 6,0 & 14,9 & 8,8 \\
\hline $4-7$ & 16,4 & 29,3 & 19,1 \\
\hline $8-10$ & 18,0 & 22,6 & 29,2 \\
\hline $11-14$ & 44,7 & 26,8 & 38,7 \\
\hline \multirow{2}{*}{$15+$} & 14,9 & 6,5 & 4,6 \\
\hline & & $\mathrm{p}<0,0001$ & \\
\hline \multicolumn{4}{|c|}{ Renda domiciliar per capita (quintis) } \\
\hline $1^{0}$ (menor) & 10,0 & 21,9 & 44,2 \\
\hline $2^{0}$ & 17,6 & 22,0 & 21,7 \\
\hline $3^{0}$ & 20,8 & 21,1 & 14,3 \\
\hline $4^{0}$ & 24,9 & 19,5 & 11,3 \\
\hline $5^{0}$ (maior) & 26,7 & $\begin{array}{r}15,5 \\
\mathrm{p}<0,0001\end{array}$ & 8,6 \\
\hline \multicolumn{4}{|l|}{ Macro-região } \\
\hline Sudeste & 70,0 & 61,7 & 62,8 \\
\hline Sul & 14,5 & 12,8 & 9,1 \\
\hline \multirow[t]{2}{*}{ Nordeste } & 15,5 & 25,5 & 28,1 \\
\hline & & $\mathrm{p}<0,0001$ & \\
\hline \multicolumn{4}{|c|}{ Número de doenças crônicas } \\
\hline 0 & 80,9 & 80,4 & 83,5 \\
\hline 1 & 15,6 & 15,3 & 12,7 \\
\hline \multirow[t]{2}{*}{$2+$} & 3,5 & 4,4 & 3,8 \\
\hline & & $\mathrm{p}<0,0006$ & \\
\hline
\end{tabular}


colaridade, renda domiciliar per capita e residir na região sul do país foram positivamente associados à visita ao dentista. Após realizar o ajuste por todas as variáveis, aqueles que estavam inseridos no trabalho sem proteção social e desempregados permaneceram com menor probabilidade de ter consultado o dentista nos últimos três anos (Tabela 4).

$\mathrm{Na}$ Tabela 5, são apresentadas algumas características da utilização de serviços e da cobertura por plano de saúde segundo a situação no mercado de trabalho em 2008. Em todas as situações no mercado de trabalho, a maioria dos homens costumava procurar o mesmo serviço de saúde quando necessitava de atendimento, sendo esse percentual um pouco menor entre aqueles inseridos no trabalho sem proteção social. Quanto ao tipo de serviço que costumavam procurar, verificou-se que mais de $61 \%$ dos que esta- vam desempregados ou inseridos no trabalho sem proteção social procuravam o centro de saúde e entre os que tinham proteção social no trabalho, $34 \%$ procuravam o centro de saúde, $29 \%$ o consultório particular e $28 \%$, ambulatórios ou consultórios de empresas, de sindicato, de clínicas e de hospitais. A cobertura por plano de saúde chegou a 47,7 dos ocupados com proteção social e quase $17 \%$ dos desempregados e trabalhadores sem proteção social. Vale ressaltar que de $78 \%$ dos planos de saúde são obtidos com a interveniência do empregador (dados não mostrados).

\section{Discussão}

Nossos resultados identificaram diferenciais na utilização dos serviços de saúde, em especial na realização de consulta médica e visita ao dentista,

Tabela 3. Prevalência de consulta médica nos últimos 12 meses, Razão de Prevalências segundo características sócio-demográficas e número de doenças crônicas em homens residentes em oito regiões metropolitanas, PNAD 2008.

\begin{tabular}{|c|c|c|c|}
\hline & $\%$ & RP IC 95\% & RP IC 95\% ajustado \\
\hline \multicolumn{4}{|c|}{ Situação mercado de trabalho } \\
\hline Com proteção social & 62,0 & 1,00 & 1,00 \\
\hline Sem proteção social & 49,6 & $0,80(0,77-0,83)$ & $0,83(0,81-0,85)$ \\
\hline Desempregado & 50,4 & $0,81(0,78-0,85)$ & $0,88(0,85-0,92)$ \\
\hline \multicolumn{4}{|l|}{ Faixa etária } \\
\hline $15-24$ & 50,6 & 1,00 & 1,00 \\
\hline $25-34$ & 54,2 & $1,07(1,03-1,11)$ & $1,02(0,98-1.06)$ \\
\hline $35-44$ & 58,6 & $1,16(1,12-1,19)$ & $1,08(1,04-1,11)$ \\
\hline $45-54$ & 62,7 & $1,24(1,19-1,29)$ & $1,09(1,05-1,14)$ \\
\hline $55-64$ & 68,7 & $1,36(1,31-1,40)$ & $1,14(1,09-1,19)$ \\
\hline \multicolumn{4}{|l|}{ Escolaridade } \\
\hline $0-3$ & 50,9 & 1,00 & 1,00 \\
\hline $4-7$ & 54,1 & $1,06(1,01-1,12)$ & $1,08(1,02-1,14)$ \\
\hline $8-10$ & 54,4 & $1,07(1,00-1,13)$ & $1,09(1,03-1,16)$ \\
\hline $11-14$ & 58,9 & $1,15(1,06-1,26)$ & $1,14(1,06-1,23)$ \\
\hline $15+$ & 68,2 & $1,34(1,23-1,46)$ & $1,17(1,10-1,25)$ \\
\hline \multicolumn{4}{|c|}{ Renda domiciliar per capita (quintis) } \\
\hline $1^{0}$ (menor) & 49,4 & 1,00 & 1,00 \\
\hline $2^{0}$ & 53,6 & $1,08(1,03-1,14)$ & $1,04(1,00-1,09)$ \\
\hline $3^{0}$ & 55,9 & $1,13(1,05-1,21)$ & $1,06(1,01-1,12)$ \\
\hline $4^{0}$ & 58,7 & $1,19(1,11-1,27)$ & $1,10(1,04-1,15)$ \\
\hline $5^{0}$ (maior) & 67,3 & $1,36(1,27-1,46)$ & $1,18(1,25-1,24)$ \\
\hline \multicolumn{4}{|l|}{ Macro-região } \\
\hline Sudeste & 57,4 & 1,00 & 1,00 \\
\hline Sul & 60,2 & $1,05(1,01-1,10)$ & $1,04(1,00-1,08)$ \\
\hline Nordeste & 55,5 & $0,97(0,92-1,02)$ & $1,05(1,00-1,10)$ \\
\hline \multicolumn{4}{|c|}{ Número de doenças crônicas } \\
\hline 0 & 52,5 & 1,00 & 1,00 \\
\hline 1 & 76,5 & $1,46(1,42-1,49)$ & $1,41(1,37-1,45)$ \\
\hline $2+$ & 85,7 & $1,63(1,58-1,69)$ & $1,58(1,53-1,63)$ \\
\hline
\end{tabular}


entre homens residentes em regiões metropolitanas de acordo com a situação no mercado de trabalho e apontaram também que esses diferen- ciais mantiveram-se praticamente inalterados nos períodos estudados. A menor utilização desses serviços entre desempregados e trabalhadores sem

Tabela 4. Prevalência de visita ao dentista há menos de três anos, Razão de Prevalências segundo características sócio-demográficas em homens residentes em oito regiões metropolitanas, PNAD 2008.

\begin{tabular}{|c|c|c|c|}
\hline & $\%$ & RP IC 95\% & RP IC 95\% ajustado \\
\hline \multicolumn{4}{|c|}{ Situação mercado de trabalho } \\
\hline Com proteção social & 77,8 & 1,00 & 1,00 \\
\hline Sem proteção social & 61,2 & $0,79(0,77-0,81)$ & $0,87(0,86-0,89)$ \\
\hline Desempregado & 68,5 & $0,88(0,84-0,92)$ & $0,95(0,92-0,99)$ \\
\hline \multicolumn{4}{|l|}{ Faixa etária } \\
\hline $15-24$ & 77,6 & 1,00 & 1,00 \\
\hline $25-34$ & 76,9 & $0,99(0,98-1,01)$ & $0,95(0,94-0,97)$ \\
\hline $35-44$ & 72,1 & $0,93(0,91-0,95)$ & $0,93(0,91-0,94)$ \\
\hline $45-54$ & 65,3 & $0,84(0,81-0,88)$ & $0,83(0,80-0,85)$ \\
\hline $55-64$ & 58,5 & $0,75(0,72-0,79)$ & $0,77(0,74-0,81)$ \\
\hline \multicolumn{4}{|l|}{ Escolaridade } \\
\hline $0-3$ & 45,6 & 1,00 & 1,00 \\
\hline $4-7$ & 57,3 & $1,27(1,20-1,35)$ & $1,22(1,15-1,28)$ \\
\hline $8-10$ & 70,3 & $1,56(1,48-1,64)$ & $1,39(1,33-1,46)$ \\
\hline $11-15$ & 81,3 & $1,80(1,71-1,90)$ & $1,51(1,44-1,58)$ \\
\hline $15+$ & 90,9 & $2,01(1,91-2,13)$ & $1,62(1,55-1,70)$ \\
\hline \multicolumn{4}{|c|}{ Renda domiciliar per capita (quintis) } \\
\hline $1^{0}$ (menor) & 55,7 & 1,00 & 1,00 \\
\hline $2^{0}$ & 64,2 & $1,15(1,11-1,20)$ & $1,10(1,06-1,15)$ \\
\hline $3^{0}$ & 69,5 & $1,25(1,21-1,29)$ & $1,17(1,14-1,20)$ \\
\hline $4^{0}$ & 76,6 & $1,37(1,32-1,43)$ & $1,25(1,20-1,30)$ \\
\hline $5^{0}$ (maior) & 87,8 & $1,57(1,51-1,64)$ & $1,35(1,29-1,41)$ \\
\hline \multicolumn{4}{|l|}{ Macro-região } \\
\hline Sudeste & 71,5 & 1,00 & 1,00 \\
\hline Sul & 78,2 & $1,09(1,02-1,18)$ & $1,10(1,05-1,14)$ \\
\hline Nordeste & 69,9 & $0,98(0,92-1,04)$ & $1,08(1,03-1,12)$ \\
\hline
\end{tabular}

Tabela 5. Características de acesso aos serviços de saúde segundo a situação no mercado de trabalho em homens residentes em oito regiões metropolitanas brasileiras, PNAD 2008.

\begin{tabular}{lccc}
\hline & \multicolumn{2}{c}{ Situação no mercado de trabalho } \\
\cline { 2 - 4 } & Com proteção social & Sem proteção social & Desempregado \\
\hline Procura o mesmo serviço de saúde & & & \\
$\quad$ Sim & 68,1 & 64,6 & 68,6 \\
Não & 31,9 & 35,4 & 32,9 \\
Tipo de serviço que procura & & & 61,8 \\
Centro de Saúde & 34,1 & 61,9 & 13,3 \\
Consultório particular & 29,3 & 12,3 & 16,1 \\
Ambulatórios ${ }^{1}$ & 28,1 & 16,1 & 7,7 \\
Emergência & 7,3 & 7,7 & 1,1 \\
Outros & 1,2 & 1,5 & 83,1 \\
Plano de saúde & & 83,4 & 16,9 \\
Não & 52,3 & 16,6 & \\
Sim & 47,7 & & \\
\hline
\end{tabular}

${ }^{1}$ Ambulatório: ambulatório ou consultório de empresa ou sindicato, ambulatório ou consultório de clínica e ambulatório ou consultório de hospital. ${ }^{2}$ Outros: farmácia, agente comunitário de saúde e outro tipo de serviço 
proteção social não pode ser atribuída às diferenças nas necessidades de saúde mensuradas pelo número de doenças crônicas, nem às demais características sócio-demográficas investigadas.

Embora tenha havido aumento no uso dos serviços odontológicos, como mostra a análise comparativa das PNADs de 1998, 2003 e $2008^{25}$, somente cerca de $40 \%$ dos brasileiros relataram consulta odontológica nos últimos 12 meses em 2008 , enquanto quase $70 \%$ informaram consulta médica nos últimos 12 meses. Essa mesma análise mostra também que as desigualdades de renda no uso de serviços odontológicos são mais acentuadas do que as relacionadas à utilização de consultas médicas ${ }^{25}$. Em nosso estudo, as associações entre situação no mercado de trabalho e visita ao dentista há menos de três anos foram menos acentuadas do que as associações com consulta médica nos últimos 12 meses. Entretanto, essas associações não são diretamente comparáveis uma vez que o tempo de referência utilizado para investigar a realização de pelo menos uma visita ao dentista foi de três anos e o de pelo menos uma consulta médica de 12 meses. Dessa forma, o critério utilizado para mensurar a utilização dos serviços odontológicos tende a reduzir os diferenciais.

Vale ressaltar que no presente estudo as magnitudes das associações entre trabalho e uso dos serviços investigados são mais acentuadas entre os trabalhadores sem proteção social do que entre os desempregados. Embora as duas situações sejam desfavoráveis para a qualidade de vida e saúde, a inserção em trabalhos precários apresenta adicionalmente a limitação de tempo para cuidar da saúde e em muitos casos o controle do empregador.

É importante ressaltar que a hospitalização parece não ser influenciada pela situação no mercado de trabalho. Analise de dados da PNAD1998 já sugeria que a renda e a educação tinham pouca importância relativa na explicação da ocorrência de internações hospitalares, embora adultos de cor branca tenham tido maior chance de hospitalização ${ }^{26}$. Estudo mais recente, realizado em Pelotas, não verificou associação entre renda e ocorrência de hospitalização em homens e mulheres de 20 a $69 \operatorname{anos}^{27}$. Apesar do relato de doenças crônicas ser mais frequente entre os trabalhadores sem proteção social e entre os que têm proteção social, não foram verificados diferenciais no acesso à hospitalização, o que pode ser atribuído ao desempenho do SUS na garantia de acesso à internação hospitalar.

A utilização e o padrão de uso dos serviços de saúde entre indivíduos empregados e desempre- gados, e ainda segundo as diferentes modalidades de inserção no trabalho, são pouco abordados nos estudos sobre o assunto. Estudos realizados nas décadas de 80 e 90 investigaram o efeito do fechamento de fábricas sobre o uso dos serviços de saúde entre os que foram demitidos enquanto outros se dedicaram a comparar a utilização dos serviços de saúde entre empregados e desempregados. Em geral, esses estudos verificaram incremento do uso entre os desempregados em países com acesso universal ao sistema de saúde ${ }^{28}$. No entanto, esses resultados são controversos. Pesquisas mais recentes indicam uma associação negativa entre desemprego e utilização dos serviços de saúde. Análise baseada em um inquérito realizado na Suécia, em 2001, sugere que apesar de relatar pior situação de saúde, os desempregados tinham menor probabilidade de consultar um médico e maior chance de não encontrar os cuidados necessários ${ }^{29}$. Outro inquérito, em amostra representativa da população adulta da Croácia, em 2003, mostrou que os desempregados, inclusive aqueles que apresentam doenças cardiovasculares e metabólicas, utilizavam menos os serviços preventivos de saúde do que os empregados ${ }^{30}$. A variação do padrão de uso segundo o tempo de desemprego pode ser uma potencial explicação para essas diferenças. É possível que indivíduos recém desempregados utilizem mais os serviços até pela maior disponibilidade gerada pelo desemprego. Já os que estão desempregados por período mais longo e sem perspectivas podem utilizar menos os serviços de saúde como reflexo do desalento e de uma menor motivação para cuidar da saúde.

Com as mudanças no trabalho e nas relações de emprego, a simples comparação entre empregados versus desempregados pode ocultar diferenças relevantes em saúde. Identificamos um estudo finlandês que investigou a utilização de serviços de saúde entre trabalhadores com contratos permanentes e temporários, com jornada integral ou parcial de trabalho, e desempregados de curta e de longa duração em amostra da população de 20 e 54 anos. Trabalhadores inseridos em empregos precários e desempregados utilizavam menos os serviços de saúde, inclusive os serviços odontológicos, do que aqueles com contrato permanente, independentemente de outras características sociodemográficas, da autoavaliação do estado de saúde e da presença de doenças ${ }^{31}$. No Brasil, Travassos et al..$^{32}$ estudaram a influência da posição no mercado de trabalho no consumo de serviços de saúde em homens e mulheres participantes da PNAD de 1998. Verifi- 
caram que homens empregados sem carteira assinada, autônomos e desempregados que apresentavam limitações nas atividades habituais por motivo de saúde haviam utilizado menos os serviços de saúde nos últimos 15 dias do que os empregados com carteira. Esses estudos corroboram os resultados do presente estudo.

As circunstâncias que envolvem a procura individual por serviço de saúde são complexas. Segundo o modelo proposto por Andersen e Newman $^{33}$, de utilização desses serviços é influenciada pelas necessidades objetivas e subjetivas de saúde; pelas características individuais como idade, sexo e escolaridade; e por fatores que facilitam a utilização, como renda, cobertura por plano e etc.

Como podemos ver, não há grandes discrepâncias na frequência do tipo de serviço de saúde que os grupos comparados no presente estudo costumam procurar. O que chama a atenção é a diferença segundo o tipo procurado: enquanto mais de $60 \%$ dos desempregados e dos trabalhadores sem proteção social procuram o centro de saúde, apenas 34\% daqueles com proteção social procuram este serviço. Esses últimos parecem contar com uma rede de oferta mais diversificada, possivelmente influenciada pela maior frequência de plano de saúde, vinculada, em grande parte, ao próprio contrato de trabalho. Além disso, trabalhadores com contratos formais de trabalho, sobretudo os que trabalham em grandes empresas, têm acesso aos serviços de saúde ocupacional das empresas e podem, contratualmente, se ausentar do trabalho mediante atestado médico. O mesmo não ocorre com os trabalhadores informais, que não estão protegidos pela legislação trabalhista. Adicionalmente, grande parte dos centros de saúde funciona em horário comercial, o que dificulta o acesso por parte dos trabalhadores.

Vale ainda ressaltar que a saúde é um importante fator de controle para o emprego e a permanência no trabalho. Trabalhadores que se ausentam por motivo de saúde têm maior probabilidade de serem despedidos, e este risco é ainda mais exacerbado no trabalho informal, pela ausência de garantias trabalhistas. Este fenômeno, conhecido como efeito do trabalhador sadio, explica em parte, a melhor condição de saúde dos que trabalham e pode explicar a menor procura de serviços dos que estão em empregos mais precários.

A condição de desempregado é estigmatizante e socialmente penalizada. Isto pode acarretar resistência do desempregado em utilizar serviços de saúde, em especial as consultas médicas, uma vez que reconhecer-se doente vulnerabilizaria ainda mais sua condição perante a família e seu núcleo social, e adicionalmente pode também influenciar negativamente sua empregabilidade ${ }^{31}$.

Como visto em nossos resultados e em outros estudos, identificou-se aqui diferenciais na utilização dos serviços de saúde estudados segundo outros indicadores de posição sócio-econômica: a utilização de serviços é menor entre os que apresentam menor escolaridade e renda. Os diferenciais regionais observados nessa população merecem particular atenção, pois os resultados presentes sugerem que homens pertencentes à população economicamente ativa (PEA), na região sudeste, realizam menos consultas médicas e odontológicas que nas regiões Sul e Nordeste. Estes resultados diferem de estudos brasileiros com a população geral ${ }^{34}$. A presente análise apresenta, como diferença em relação aos estudos com populações gerais, o recorte amostral (PEA masculina) e o ajuste por situação de trabalho. Entretanto, os dados existentes e a falta de estudos anteriores sobre o tema nesta população não nos permite apontar potenciais explicações para essas diferenças.

As desigualdades observadas nesse estudo se tornam ainda mais relevantes e injustas quando se verifica que são independentes da presença de doenças crônicas, responsáveis pela maior carga de enfermidades na atualidade. Vale ressaltar que estas incluem a hipertensão e o diabetes, que necessitam de acompanhamento frequente e uso de medicação regular. Como muitas permanecem silenciosas por longo tempo, é possível que a prevalência e o número podem estar subestimados, especialmente entre os trabalhadores informais e desempregados, uma vez que utilizam menos os serviços de saúde.

É sabido que os indivíduos que ingressam no emprego, sobretudo naqueles com contratos formais, são os mais saudáveis, e que sua condição de saúde, inclusive, é um fator que influencia sua permanência no emprego. Por outro lado, se desempregados têm piores condições de saúde do que os que trabalham, deveriam utilizar mais os serviços de saúde para fazer frente a sua maior necessidade. Entretanto, como vimos, eles os utilizam menos, o que pode piorar sua saúde e comprometer sua inserção no trabalho. Ou seja, a desigualdade na utilização nos serviços de saúde entre desempregados parece fazer parte de um circulo socialmente perverso.

Este estudo contribui para a discussão sobre desigualdade sociais em saúde ao investigar a influência das novas modalidades de inserção no 
mercado de trabalho sobe a utilização de serviços de saúde. Os resultados sugerem que a estrutura social e econômica, que estabelece diferentes grupos sociais com condições de saúde desiguais, também se faz presente no uso deles, o que pode contribuir para reproduzir e acentuar as desigualdades existentes. O Sistema Único de Saúde, que representa um enorme avanço em termos de políticas públicas no Brasil, ainda não foi capaz de fazer face à desigualdade na utilização de serviços, em especial no uso de serviços odontológicos e consultas médicas, e na população masculina economicamente ativa. Conhecer as barreiras e aumentar a oferta de serviços com perfil de funcionamento adequado para quem trabalha é fundamental para tentar reduzir as iniquidades observadas. Por outro lado, promover a inserção no trabalho com garantias dos direitos sociais pode contribuir para a melhoria da saúde pública.

\section{Colaboradores}

L Giatti e SM Barreto participaram igualmente de todas as etapas de elaboração do artigo.

\section{Agradecimentos}

L. Giatti é bolsista do Programa de Apoio a Projetos Institucionais com a Participação de RecémDoutores, da Coordenação de Aperfeiçoamento de Pessoal de Nível Superior (PRODOC-CAPES). Sandhi Maria Barreto é bolsista de produtividade em pesquisa do Conselho Nacional de Desenvolvimento Científico e Tecnológico (CNPq; processo no. 300159/99-4).

\section{Referências}

1. Word Health Organization (WHO). Commission on Social Determinants of Health. Employment and health inequalities. Employment Conditions Knowledge Network (EMCONET). Final Report, 20 September 2007.

2. Krieger N. Workers Are People Too: Societal Aspects of Occupational Health Disparities - An Ecosocial Perspective. Am J Ind Med 2010; 53(2): 104-115.

3. Lundin A, Lundberg I, Hallsten L, Ottosson J, Hemmingsson T. Unemployment and mortality-a longitudinal prospective study on selection and causation in 49321 Swedish middle-aged men. J Epidemiol Community Health 2010; 64(1):22-28.

4. Giatti L, Barreto SM, César CC. Unemployment and self-rated health: neighborhood influence. Soc Sci Med 2010; 71(4):815-823.

5. Voss M, Nylén L, Floderus B, Diderichsen F, Terry RD. Unemployment and early cause-specific mortality: a study based on the Swedish Twin Registry. Am J Public Health 2004; 94(12): 2155-2161

6. Artacoz L, Benach J, Borrell C, Cortés I. Unemployment and mental health: understanding the interactions among gender, family roles and social class. Am J public Heatlh 2004; 94(1):82-88.

7. Schunck R, Rogger BG. Unemployment and its association with health-relevant actions: investigating the role of time perspective with German census data. Int J Public Health 2010; 55(4):271-278. doiI 10.1007/s00038-009-0075-1 
8. Reine M, Hammarström A. Does the association between ill health and unemployment differ between young people and adults? Results from a 14-year folow-up study with a focus on psychological health and smoking. Public Health 2004; 118(5):337-345.

9. Landsbergis PA. Assessing the contribution of working conditions to socioeconomic disparities in health: a commentary. Am J Ind Medicine 2010; 53(2):95-103.

10. Lahelma E, Laaksonen M, Aittomäki A. Occupational class inequalities in health across employment sectors: the contribution of working conditions. Int Arch Occup Environ Health 2009; 82(2):185-190.

11. Samia R, Ostry A, LaMontagne D. Job stress and other working condictions: a relationships with smoking beahaviors in a representative of working Australians. Am J Ind Med 2007; 50(8):584-596.

12. Clougherty JE, Souza K, Cullen MR. Work and its role in shapping the social gradient in health. Ann N Y Acad Sci 2010; 1186:102-124.

13. Virtanen P, Liukkonem V, Kivimäki M, Koskenvuo M. Health inequality in the workforce: the labour market core-periphery structure. Int J Epidemiol 2003; 32(6):1015-1021.

14. Giatti L, Barreto SM, César CC. Social protection at work, unemployment and health in Brazilian metropolitan areas, 1998 and 2003. Cad Saude Publica 2008; 24(10):2396-406.

15. Kim M-H, Kim C-Y, Park J-K, Kawachi. Is precarious employment damaging to self-rated health? Results of propensity score matching methods, using longitudinal data in South Korea. Soc Sci Med 2008; 67(12):1982-1994.

16. Quesnel-Vallée A, DeHaney S, Ciampi A. Temporary work and depressive symptoms: A propensity score analysis. Soc Sci Med 2010; 70(12):1982-1987.

17. Natti J, Kinnunen U, Makikangas A, Mauno S. Type of employment relationship and mortality: prospective study among Finnish employees in 1984-2000. Eur J Public Health 2009; 19(2):150-156.

18. Kawachi I, Subramanian SV, Almeida-Filho N. A glossary for health inequalities. J Epidemiol Community Health 2002; 56:647-652.

19. Comissão Nacional sobre Determinantes Sociais da Saúde (CNDSS). As causas das iniquidades em saúde no Brasil. Rio de Janeiro: CNDSS; 2008. (Relatório final). [acessado $2011 \mathrm{fev} \mathrm{10].} \mathrm{Disponível} \mathrm{em:} \mathrm{www.}$ cndss.fiocruz.br/pdf/home/relatorio.pdf

20. Williams A. Thinking about equity in health care. $J$ Nurs Manag 2005;13(5):397-402.

21. Barata RB, de Almeida MF, Montero CV, da Silva ZP. Gender and health inequalities among adolescentes and adults in Brazil, 1998. Rev Panam Salud Publica 2007; 21(5):320-327.

22. Pinheiro, RS, Viacava F, Travassos C, Brito AS. Gênero, morbidade, acesso e utilização de serviços de saúde no Brasil. Cien Saude Colet 2002; 7(4):687707.

23. Menendez M, Benach J, Muntaner C, Amable M, O'Campo P. Is precarious employment more damaging to women's health than men's? Soc Sci Med 2007; 64(4):776-781.
24. Silva PLN, Pessoa DGC, Lila MF. Análise estatística de dados da PNAD: incorporando a estrutura do plano amostral. Cien Saude Colet 2002; 7(4):659-670.

25. Viacava F. Acesso e uso de serviços de saúde pelos brasileiros. Radis 96 2010; agosto:2010. [acessado 2011 fev 05]. Disponível em: www4.ensp.fiocruz.br/ radis/96/pdf/radis_96.pdf

26. Castro MSM, Travassos C, Carvalho MS. Fatores associados às internações hospitalares no Brasil. Cien Saude Colet 2002; 7(4):795-811.

27. Costa JSD, Reis MC, Silveira Filho CV, Linhares RS, Piccinini F, Fantinel E Prevalência de internação hospitalar e fatores associados em Pelotas, RS. Rev Saude Pública 2010; 44(5):923-933.

28. Jin R, Shah CP, Svoboda TJ The impact of unemployment on health: a review of the evidence. Can Med Assoc J 1995; 153(5):539-540.

29. Ahs AMH, Westerling R, Health care utilization among persons who are unemployed or outside the labour force. Health Policy 2006; 78(2-3):178-193.

30. Luka Vonèina L, Pristaš I, Mastilica M, Polašek $O$, Šošiæ Z, Stevanoviæ R. Use of Preventive Health Care Services among the Unemployed in Croatia. Croat Med J 2007; 48(5):667-674

31. Virtanen P, Kivimaki M, Vahtera J, Koskenvuo M. Employment status and differences in the one-year coverage of phisycians vissts: need or unequal access to services? BMC Health Services Research 2006, 6:123. Doi:10.1186/1472.6963/6/123. [acesso $2011 \mathrm{fev}$ 10]. Disponível em: www.cndss.fiocruz.br/pdf/home/ relatorio.pdf

32. Travassos C, Viacava F, Pinheiro R, Brito A. Utilização dos serviços de saúde no Brasil: gênero, características familiares e condição social. $\mathrm{Rev} P \mathrm{~Pa}$ nam Salud Publica 2002;11(5-6):365-373.

33. Andersen R, Newman JF: Societal and individual determinants of medical care utilization in the United States. Milbank Mem Fund Q Health Soc 1973, 51:95-124.

34. Travassos C, Oliveira E.X.G., Viacava F. Desigualdades geográficas e sociais no acesso aos serviços de saúde no Brasil: 1998 e 2003. Cien Saude Colet 2006; 11(4):975-986.

Artigo apresentado em 11/02/2010

Aprovado em 02/05/2010

Versão final apresentada em 08/06/2011 